\title{
Diagnóstico de la ansiedad utilizando Sistemas Expertos con el test psicológico "Inventario de Ansiedad de Beck"
}

\author{
Anxiety diagnosis using Expert System with the psychological test "Beck Anxiety Inventory"
}

Fecha de recepción y aceptación: 2 de diciembre de 2019 y 22 de mayo de 2020

DOI: $10.46583 /$ nereis 2020.12.611

\section{Elizabeth Vilcacuri Huamani ${ }^{1 *}$ y Laura Liz Rodriguez Solis ${ }^{1}$}

${ }^{1}$ Facultad de Ingeniería de Sistemas e Informática. Universidad Nacional Mayor de San Marcos.

${ }^{*}$ Correspondencia: Universidad Nacional Mayor de San Marcos. Calle Germán Amézaga, n. ${ }^{\circ} 375$ - Edificio Jorge Basadre. Ciudad Universitaria. Lima 1., Lima. Perú. E-mail: elizabeth.vilcacuri@unmsm.edu.pe

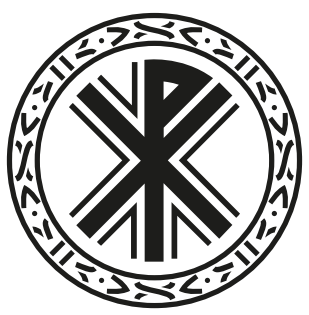

\section{RESUMEN}

La salud mental en el Perú es uno de los graves problemas que afecta a nuestra sociedad, uno de ellos es la ansiedad, que puede presentarse como una respuesta normal al estrés, pero cuando no se puede controlar puede afectar e interferir en la vida cotidiana de las personas, por ello es muy importante que lo detecten a tiempo, yendo al psicólogo para su evaluación respectiva. El test psicológico "Inventario de Ansiedad de Beck" permite obtener el nivel de ansiedad y junto con el análisis del psicólogo se obtiene el diagnóstico, este proceso requiere de un tiempo largo, por lo que muchas veces no es aplicado el test en el Consultorio Psicológico de la Universidad Nacional Mayor de San Marcos. La implementación de un sistema experto mediante la metodología CommonKADS, utilizando las herramientas NetBeans para la interfaz gráfica, el programa CLIPS para la base de conocimiento y MySQL para la base de datos, permitirá reducir el tiempo en la obtención del diagnóstico de ansiedad. En este estudio se confirmará la confiabilidad del sistema en un $90 \%$ por lo que se concluye que el Sistema Experto mejorará el tiempo de respuesta en la obtención del diagnóstico de la ansiedad, ofreciendo un servicio más rápido a los pacientes universitarios, siendo de gran ayuda para el psicólogo y beneficiando al Consultorio Psicológico de la universidad con el aumento de frecuencia de pacientes.

PALABRAS CLAVE: ansiedad, sistemas expertos, psicología, inteligencia artificial.

\section{ABSTRACT}

Mental health in Peru is one of the most serious problems that affects our society, one of them being anxiety, which can be present as a normal response to stress. But when it cannot be controlled, it can affect and interfere in people's daily lives, so it is very important to detect it in time and go to counseling to get assessment. The psychological test "Beck's Anxiety Inventory" allows us to obtain the patient's level of anxiety, and together with the analysis of the psychologist the diagnosis is thus obtained; this process takes a long time, and this is why many times the test is not applied in the Psychological Consultory of the Universidad Nacional Mayor de San Marcos. The implementation of an Expert System through the CommonKADS methodology, 
using NetBeans tools for the graphical interface, the CLIPS program for the knowledge base and MySQL for the database, will reduce the time in which the diagnosis of anxiety is obtained. In this study we will confirm the reliability of the system by $90 \%$, so it is concluded that the Expert System will improve the response time in obtaining the diagnosis of anxiety, offering a faster service to university patients, being of great help to the psychologist and benefiting the Psychological Clinic of the university with the increased frequency of patients.

KEYWORDS: anxiety, expert systems, psychology, artificial intelligence.

\section{INTRODUCCIÓN}

La ansiedad es una emoción común de las personas frente a situaciones estresantes y de incertidumbre y la emoción cumple una función útil que permite adaptarse a la vida. Este tipo de ansiedad puede ayudar a estar alerta, a ser más productivo y a realizar el trabajo de manera más eficiente. Sin embargo, hay momentos en los que la ansiedad más que resultar ser útil se convierte en un obstáculo para llevar una vida normal. Esto ocurre cuando aparecen síntomas de ansiedad sin motivos aparentes, o bien, que el nivel de ansiedad ante un acontecimiento es totalmente desproporcionado respecto al peligro real que supone. [1]

El diagnóstico de la ansiedad es complicado, porque este trastorno psicológico puede presentar diversos síntomas que tienen que ser cuidadosamente analizados; es así que los psicólogos al realizar un análisis exhaustivo de este, invierten mucho tiempo para la obtención del diagnóstico de la ansiedad.

Por ello este presente trabajo de investigación tiene como objetivo implementar un sistema experto que permita la obtención del diagnóstico de la ansiedad usando el test psicológico Inventario de Ansiedad de Beck (BAI), con el fin de mejorar el tiempo del proceso de la evaluación psicológica, identificando el nivel de ansiedad que pueden presentar los pacientes y permitiendo la entrega rápida de los resultados.

Dicho sistema se implementará utilizando técnicas de Inteligencia Artificial como reglas Si-Entonces y con tecnologías Open Source, el sistema utilizará el conocimiento previo de un experto (Psicólogo); esto es, la información necesaria para la evaluación y la experiencia capturada en forma de reglas de inferencia, con el fin de analizar los resultados de la evaluación del test psicológico BAI y así como las respuestas de las preguntas adicionales formuladas por el psicólogo.

La importancia de este presente artículo se justifica en tres puntos principales:

Primero, este sistema experto será de gran ayuda para los psicólogos que forman parte de la clínica, ya que permitirá que el tiempo que se toman para el análisis del resultado del test psicológico BAI, así como la recopilación de la información necesaria para la evaluación, se reduzca significativamente.

Segundo, en el aspecto social, al brindar una entrega rápida de diagnósticos, el paciente podrá buscar a tiempo recomendaciones oportunas, tratamientos psicológicos, etc. que le ayuden a vencer estos problemas psicológicos, teniendo como resultado la mejora del aspecto emocional del paciente.

Tercero, en el aspecto económico, permitirá a la clínica brindar un mejor servicio a los pacientes, ya que se aplicará un nuevo mecanismo de evaluación y se entregarán de manera más rápida los diagnósticos de la ansiedad, así teniéndolos satisfechos y posibilitando el aumento de la frecuencia con la que se aplica el test psicológico BAI y por ende disminuir la posibilidad de pérdida de pacientes. 
Cabe destacar que el sistema experto a desarrollar en la clínica psicológica no busca reemplazar al especialista, sino que busca ser una herramienta de apoyo y guía.

\section{MATERIALES Y MÉTODOS}

\section{Inventario de ansiedad de Beck}

El test BAI, es el instrumento psicológico que utilizamos para poder diagnosticar la ansiedad en los estudiantes universitarios; fue desarrollado por Beck en 1988, con el objetivo de poseer un instrumento capaz de diagnosticar de manera fiable la ansiedad.

Es un inventario autoaplicado, compuesto por 21 ítems, que describen diversos síntomas de ansiedad [2].

\section{Sistema experto}

Para solucionar el problema planteado se hizo uso de un Sistema Experto, el cual se define como un conjunto de programas que tiene una base de conocimiento con la información de uno o más expertos sobre un determinado tema, utilizado para poder resolver problemas.

Arquitectura de un sistema experto

La arquitectura del sistema experto está compuesto por los siguientes componentes [3]

- Módulo de adquisición de conocimiento

- Base de conocimiento

- Base de hechos

- Motor de inferencia

- Subsistema de explicación

- Interfaz de usuario

El tipo de sistema experto que se utilizó está basado en reglas, las cuales tienen el siguiente formato:

SI - ENTONCES, (IF - THEN)

Donde:

SI (IF): Este es el antecedente, premisa, condición o situación.

ENTONCES (THEN): Esta segunda parte es el consecuente, conclusión, acción o respuesta.

En este sistema basado en reglas se utilizó el modus ponens para manipular los hechos y las reglas durante el proceso de inferencia [4]. 


\section{Metodología CommonKADS}

La metodología utilizada para el desarrollo e implementación del sistema experto fue Commonkads, la cual es una metodología para el análisis y construcción de sistemas de conocimiento donde se especifica todo el ciclo de desarrollo de sistemas expertos, comprende el modelado del conocimiento y el razonamiento que posee un experto en un dominio específico con el propósito de desarrollar un sistema que resuelva los problemas tal como lo hace el experto humano.

Se descompone en tres submodelos: el modelo del contexto, el modelo conceptual y el modelo de diseño para su construcción. Cada modelo mencionado se descompone a su vez en otros modelos más específicos [5].

Hemos aplicado la metodología de la siguiente manera:

\section{Modelo del contexto:}

Este modelo nos permitió analizar la organización, la clínica psicológica COPSI, entender el contexto y el entorno de trabajo.

Es así que este modelo nos ayudó a justificar la razón del porqué se pretende construir un Sistema Experto para implementarlo en la clínica psicológica, permitiendo identificar los problemas y oportunidades a resolver, y considerando el impacto que se producirá en la organización de la clínica psicológica.

Este se descompone en 3 submodelos más: Modelo de la organización, Modelo de las tareas, Modelo de los agentes.

\section{Modelo conceptual:}

El sistema experto que se implementó contiene las reglas basadas en el conocimiento de un experto psicólogo de la clínica COPSI que se encarga del análisis de los test BAI que se realizan ahí. Las reglas implementadas en el sistema permiten brindar un diagnóstico a los pacientes de forma inmediata y precisa.

Este se descompone en 2 submodelos más: Modelo de conocimiento, Modelo de comunicación.

\section{Reglas implementadas:}

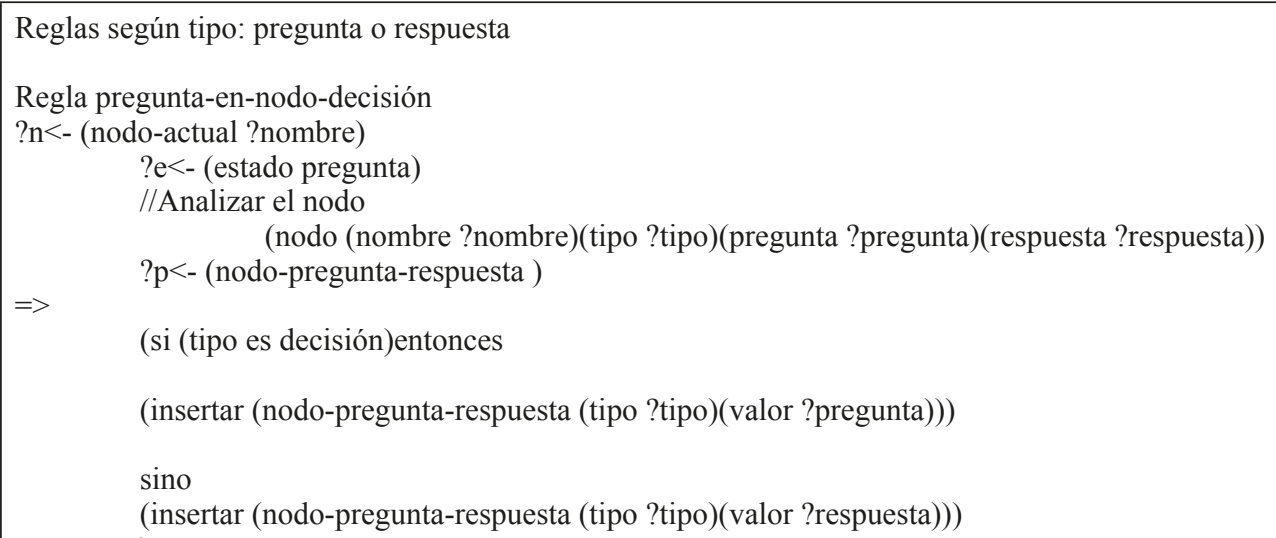




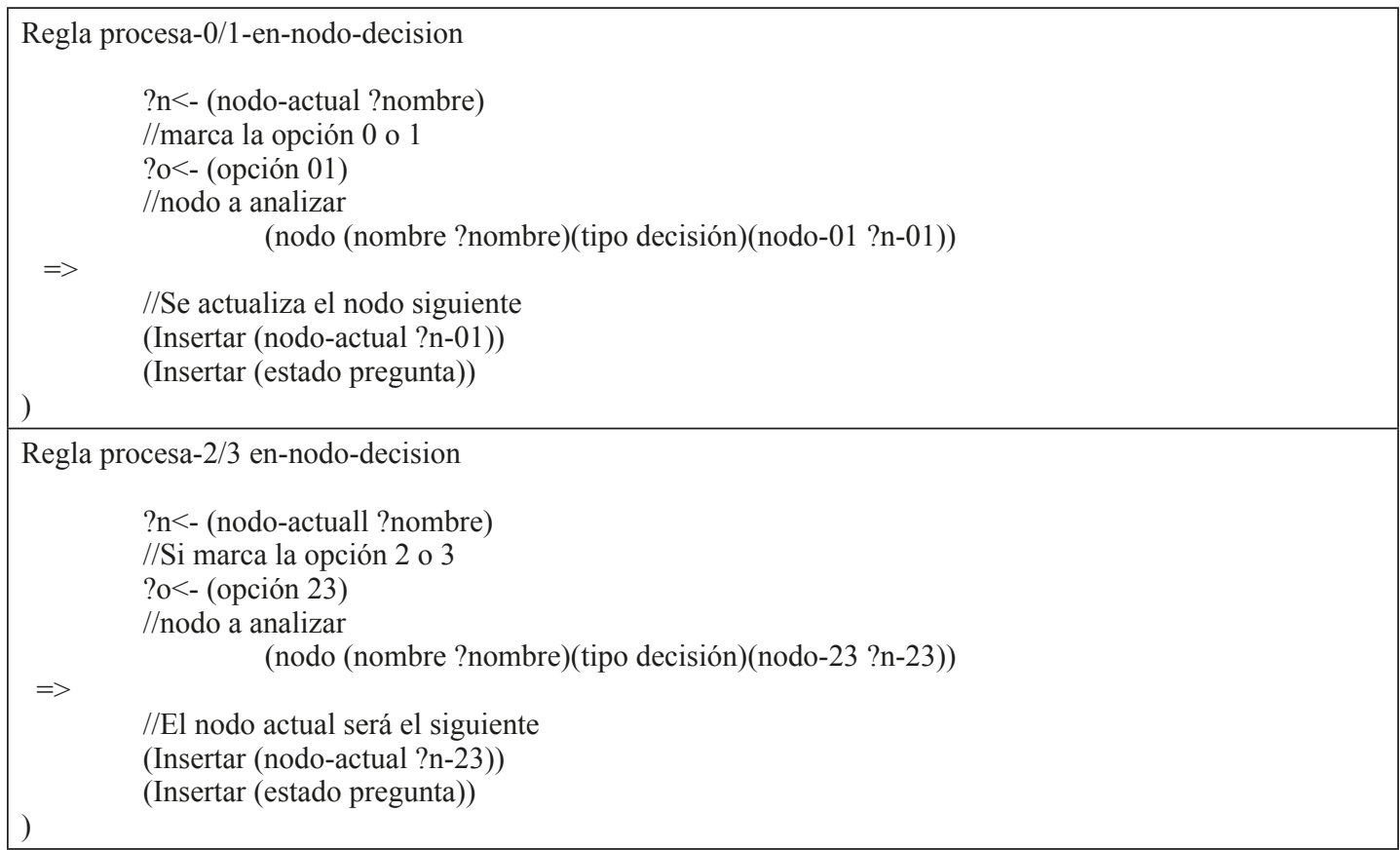

\section{Modelo de construcción:}

Para el desarrollo del sistema experto, se determinó el uso de JAVA, CLIPS y MYSQL como herramientas para el desarrollo del mismo, CLIPS, como herramienta para la implementación de la base de conocimiento del sistema y JAVA para la interfaz de usuario.

Para almacenar la información ingresada tanto del usuario como del experto, se seleccionó la base de datos MYSQL, debido a la facilidad de su configuración e instalación.

Este se descompone en 1 submodelo más: Modelo de diseño.

\section{APORTE PRÁCTICO}

\section{Diagrama solución}

En el diagrama (figura 1) se da una vista de general de la aplicación del sistema experto que proponemos, mostrando inicialmente al paciente, quien realizará su evaluación psicológica mediante el sistema experto por medio de una computadora o una tableta.

Una vez realizado el test de la evaluación psicológica, los datos ingresados y el diagnóstico proporcionado se almacenarán en un servidor el cual guardará toda la información de las evaluaciones psicológicas; esta información la maneja la clínica COPSI, con el cuidado que se requiere en estos casos.

El diagnóstico dado por el sistema experto podrá ser visto y analizado por los psicólogos de la clínica para su verificación, si es que lo requieren podrán ingresar a la información a través del sistema el cual mostrará todos los resultados dados hasta el momento, por lo que ellos viendo esos resultados podrían dar recomendaciones a los pacientes. 


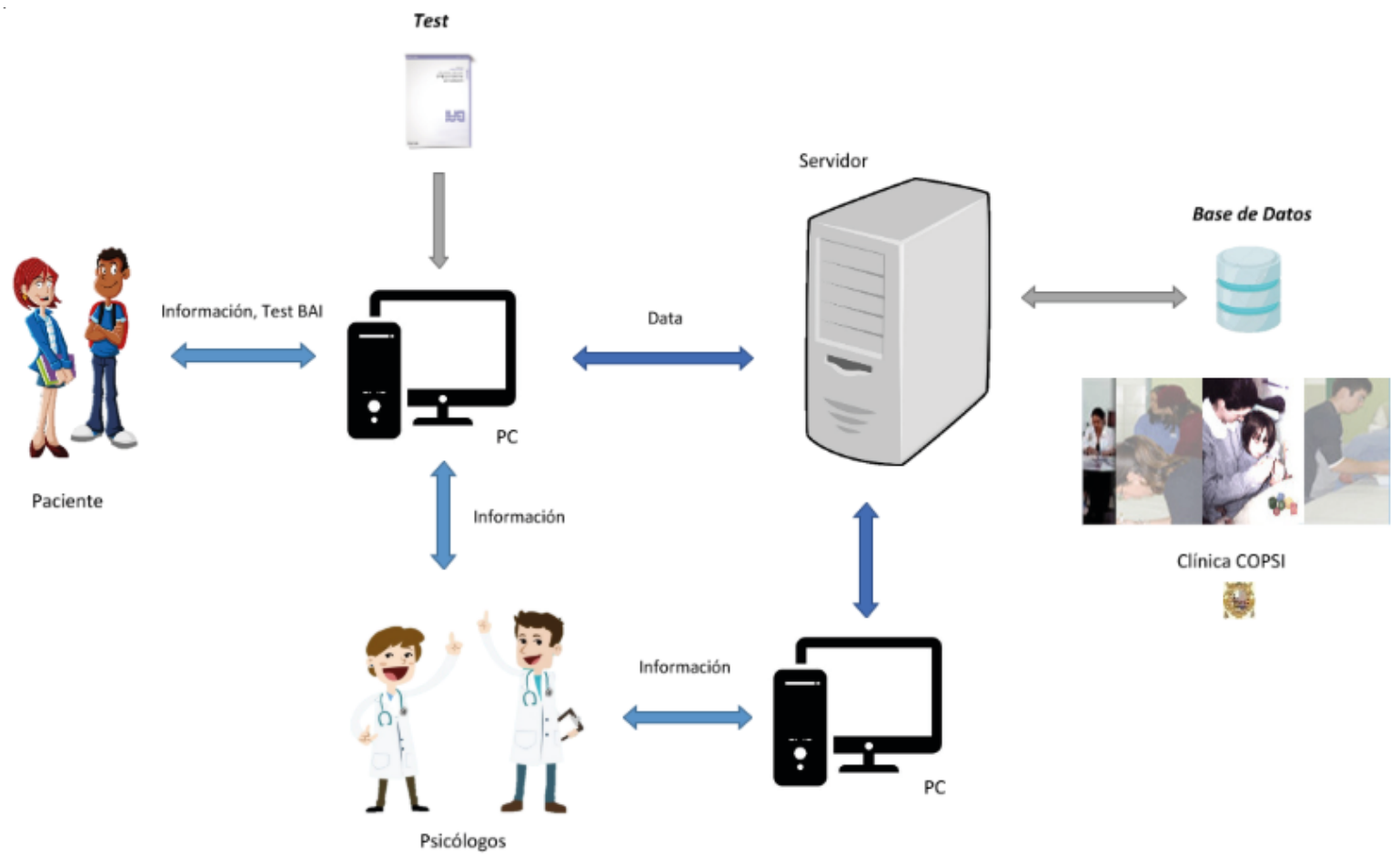

Fig. 1. Diagrama de la solución propuesta. Fuente: elaboración propia.

\section{RESULTADOS}

A continuación, se detalla cómo se realizarán las pruebas y validaciones para verificar que el sistema experto responde correctamente a los objetivos de la presente tesis.

Se presenta lo que sería la validación del software, es decir, la confiabilidad del sistema experto, se muestra una comparación de tiempos de la obtención del diagnóstico del test psicológico BAI, la medición del nivel de satisfacción del usuario tanto para paciente como para el psicólogo al utilizar el sistema experto y por último se presenta una comparación del número de pacientes que concurrirán a la clínica COPSI para la realización del TEST BAI durante los últimos meses del 2019.

La validación se realizará en base a las variables de nuestra investigación, las cuales son:

- Variable independiente:

- Sistema experto

- Sistema automatizado

- Variable dependiente:

- $\quad$ Proceso de la evaluación psicológica del test BAI 


\section{Indicadores de medición}

Se han definido indicadores en base a las variables dependientes e independientes que ayudarán a medir en qué grado se han cumplido los objetivos del trabajo de investigación.

Se plantearon los siguientes indicadores:

Sistema experto

Porcentaje de aciertos de resultados del diagnóstico del sistema experto con el experto humano.

Sistema automatizado

Porcentaje de aciertos del nivel de ansiedad según la clasificación del Test BAI.

Proceso de la evaluación psicológica del Test BAI

Tiempo de obtención del diagnóstico del test psicológico BAI.

Porcentaje de satisfacción del usuario al utilizar el sistema.

Número de pacientes que tomaron el TEST BAI durante 1 mes.

\section{Escenario de validación}

Para las pruebas y validaciones del sistema experto, se debe definir claramente la población y muestra en la que se va a aplicar.

Población: Para la realización de pruebas y validaciones se tomará en cuenta a los pacientes que asisten a la clínica COPSI.

Muestra: Se determinará una muestra representativa de la población, la cual es la cantidad total de pacientes que van a tomar el test psicológico BAI en la clínica COPSI durante un mes. Aproximadamente 10 pacientes.

\section{Procesamiento y muestra de resultados de la validación del sistema experto}

\section{Objetivo}

Agilizar el análisis y la entrega del diagnóstico del test psicológico BAI, lo cual permitirá brindar satisfacción a los pacientes, ya que tendrán conocimiento de su diagnóstico en un tiempo corto.

Variable independiente

Sistema experto.

Indicador

Porcentaje de aciertos de resultados del diagnóstico del sistema experto con el experto humano. 
Validación de la confiabilidad del sistema experto

Para comprobar que el sistema experto brinda diagnósticos correctos a los pacientes, se realizarán iteraciones en las cuales se pretende verificar que cada diagnóstico proporcionado por el sistema experto coincida o se aproxime lo mayor posible al diagnóstico dado por el Experto humano, es así que en cada iteración se realizarán los ajustes necesarios, obteniendo así al final de las iteraciones un sistema experto más preciso.

En el proceso de pruebas se realizarán tres iteraciones, pretendiendo obtener en cada iteración un resultado más preciso.

\section{Resultado}

\section{Primera iteración}

En esta primera iteración se pretende observar que, del total de los 10 casos de la muestra, el sistema detectará 7 casos que coinciden con los resultados dados por el experto, lo que arrojará un porcentaje de acierto del $70 \%$.

Tabla 1. Resultados de la primera iteración

\begin{tabular}{cccc}
\hline PACIENTE & EXPERTO & SE & COINCIDENCIA \\
\hline A & D1 & D2 & NO \\
B & D1 & D1 & SÍ \\
C & D2 & D2 & SÍ \\
D & D1 & D2 & NO \\
E & D3 & D3 & SÍ \\
F & D3 & D3 & SÍ \\
G & D1 & D1 & SÍ \\
H & D2 & D2 & SÍ \\
I & D3 & D1 & NO \\
J & D3 & D3 & SÍ \\
\hline
\end{tabular}

Fuente: elaboración propia.

\section{Segunda iteración}

Para la segunda iteración, se realizarán unos ajustes en las reglas para la obtención del diagnóstico BAI y se pretenderá observar que del total de los 10 casos, el sistema detectará 8 casos que coinciden con los resultados dados por el experto, lo que arrojará un porcentaje de acierto del $80 \%$.

Tabla 2. Resultados de la segunda iteración

\begin{tabular}{cccc}
\hline PACIENTE & EXPERTO & SE & COINCIDENCIA \\
\hline A & D1 & D2 & NO \\
B & D1 & D1 & SÍ \\
C & D2 & D2 & SÍ \\
D & D1 & D2 & NO
\end{tabular}




\begin{tabular}{cccc}
\hline PACIENTE & EXPERTO & SE & COINCIDENCIA \\
\hline E & D3 & D3 & SÍ \\
F & D3 & D3 & SÍ \\
G & D1 & D1 & SÍ \\
H & D2 & D2 & SÍ \\
I & D3 & D3 & SÍ \\
J & D3 & D3 & SÍ \\
\hline
\end{tabular}

Fuente: elaboración propia.

\section{Tercera iteración}

Para la tercera iteración, se realizarán los últimos ajustes en las reglas para la obtención el diagnóstico BAI y se pretende observar que del total de los 10 casos de la muestra, el sistema detectará 9 casos que coinciden con los resultados dados por el experto, lo que arrojará un porcentaje de acierto del $90 \%$.

Tabla 3. Resultados de la tercera iteración

\begin{tabular}{cccc}
\hline PACIENTE & EXPERTO & SE & COINCIDENCIA \\
\hline A & D1 & D1 & SÍ \\
B & D1 & D1 & SÍ \\
C & D2 & D2 & SÍ \\
D & D1 & D2 & NO \\
E & D3 & D3 & SÍ \\
F & D3 & D3 & SÍ \\
G & D1 & D1 & SÍ \\
H & D2 & D2 & SÍ \\
I & D3 & D3 & SÍ \\
J & D3 & D3 & SÍ \\
\hline
\end{tabular}

Fuente: elaboración propia.

\section{Procesamiento y muestra de resultados de la validación del sistema automatizado}

Objetivo

Agilizar el análisis y la entrega del diagnóstico del test psicológico BAI, lo cual permitirá brindar satisfacción a los pacientes, ya que tendrán conocimiento de su diagnóstico en un tiempo corto.

Variable independiente

Sistema automatizado.

\section{Indicador}

Porcentaje de aciertos del nivel de ansiedad según la clasificación del Test BAI. 
Validación de la confiabilidad del sistema automatizado

Para comprobar que el sistema automatizado identifica correctamente el nivel de ansiedad de un paciente según la clasificación del Test BAI, se realizará un análisis comparativo entre el resultado dado por el sistema y el puntaje obtenido de la realización del Test BAI de forma manual.

\section{Resultado}

Como se puede observar en la tabla, se pretende presentar un porcentaje de acierto del $100 \%$, ya que en los resultados de todas las personas que tomarán la evaluación psicológica, coincide el nivel de ansiedad dado por el sistema automatizado y el resultado del puntaje obtenido del test psicológico.

Tabla 4. Resultados del Sistema Automatizado

\begin{tabular}{cccc}
\hline PACIENTE & SISTEMA AUTOMATIZADO & CLASIFICACIÓN DEL TEST BAI & COINCIDENCIA \\
\hline A & Alta & Alta & SÍ \\
B & Baja & Baja & SÍ \\
C & Baja & Baja & SÍ \\
D & Media & Media & Sí \\
E & Media & Media & SÍ \\
F & Media & Media & Sí \\
G & Baja & Baja & SÍ \\
H & Alta & Alta & SÍ \\
I & Alta & Alta & SÍ \\
J & Alta & Alta & SÍ \\
\hline
\end{tabular}

Fuente: elaboración propia.

\section{Procesamiento y muestra de resultados de la validación del proceso de obtención del diagnóstico BAI}

Objetivo

Agilizar el análisis y la entrega del diagnóstico del test psicológico BAI, lo cual permitirá brindar satisfacción a los pacientes, ya que tendrán conocimiento de su diagnóstico en un tiempo corto.

Variable dependiente

Proceso de la evaluación psicológica del test BAI.

\section{Indicador}

Tiempo de obtención del diagnóstico del test psicológico BAI.

Validación del rápido análisis y entrega del diagnóstico del Test psicológico

Para comprobar que el sistema experto permite un rápido análisis y entrega del diagnóstico, se realizará un análisis comparativo sobre el tiempo de obtención del diagnóstico dado por el sistema experto y el tiempo de obtención del diagnóstico proporcionado por el psicólogo. 


\section{Resultado}

Como se puede apreciar en la siguiente tabla, se pretender validar que el sistema experto mejora notablemente el tiempo de obtención del diagnóstico del Test BAI, reduciéndose de horas, que empleaba el psicólogo para el análisis y entrega de diagnósticos, a segundos.

Tabla 5. Resultados del tiempo del proceso de diagnóstico

\begin{tabular}{ccc}
\hline PACIENTE & FORMA MANUAL & AUTOMÁTICO \\
\hline $\mathrm{A}$ & $2 \mathrm{~h}$ & $5 \mathrm{~s}$ \\
$\mathrm{~B}$ & $1.45 \mathrm{~h}$ & $6 \mathrm{~s}$ \\
$\mathrm{C}$ & $2 \mathrm{~h}$ & $6 \mathrm{~s}$ \\
$\mathrm{D}$ & $1.45 \mathrm{~h}$ & $5 \mathrm{~s}$ \\
$\mathrm{E}$ & $2 \mathrm{~h}$ & $4.5 \mathrm{~s}$ \\
$\mathrm{~F}$ & $1.45 \mathrm{~h}$ & $4.8 \mathrm{~s}$ \\
$\mathrm{G}$ & $2 \mathrm{~h}$ & $4.3 \mathrm{~s}$ \\
$\mathrm{H}$ & $1 \mathrm{~h}$ & $4.6 \mathrm{~s}$ \\
$\mathrm{I}$ & $1 \mathrm{~h}$ & $8.9 \mathrm{~s}$ \\
$\mathrm{~J}$ & $1.30 \mathrm{~h}$ & $4.3 \mathrm{~s}$ \\
\hline
\end{tabular}

Fuente: elaboración propia.

\section{Indicador}

Porcentaje de usuarios satisfechos al utilizar el sistema.

\section{Validación de la satisfacción del usuario al utilizar el sistema}

Para comprobar que el sistema brinda la sensación de satisfacción en los usuarios, se realizará una encuesta tanto para los pacientes como para los psicólogos, donde permite identificar qué tan satisfechos se encuentran con el sistema, si lo consideran fácil de usar, si lo ven amigable y de uso intuitivo, etc.

\section{Resultado}

Encuesta de satisfacción del sistema al psicólogo.

Como se puede apreciar en la siguiente tabla, se pretende obtener que a través de la encuesta que se realizaría a tres psicólogos de la clínica COPSI, se observará que se encuentran satisfechos con el sistema, ya que dan las máximas puntuaciones a las preguntas positivas, es decir, que están de acuerdo con dichas preguntas de la encuesta y la mínima puntuación a la preguntas negativas de la encuesta, es decir, que se encuentran en desacuerdo. 
Tabla 6: Resultados de la encuesta a los psicólogos

\begin{tabular}{|c|c|c|c|}
\hline Preguntas de la encuesta & Psicólogo A & Psicólogo B & Psicólogo C \\
\hline 1. Es de gran ayuda el sistema experto & 5 & 4 & 5 \\
\hline 2. El Sistema Experto da resultados correctos & 4 & 4 & 4 \\
\hline 3. Los mensajes que aparece en el sistema son de fácil comprensión & 4 & 5 & 4 \\
\hline 4. El sistema es muy fácil de utilizar & 5 & 5 & 5 \\
\hline 5. El sistema es fácil de aprender & 5 & 5 & 5 \\
\hline 6. Recomendaría el uso del sistema para otras clínicas psicológicas & 5 & 4 & 5 \\
\hline 7. El sistema toma más tiempo que el trabajo manual & 1 & 1 & 1 \\
\hline 8. Me ayuda en mi trabajo diario & 4 & 4 & 4 \\
\hline 9. Tuve problemas en utilizar el sistema & 1 & 1 & 1 \\
\hline 10. Puedo encontrar fácilmente las funciones que ofrece el sistema & 4 & 4 & 4 \\
\hline
\end{tabular}

Fuente: elaboración propia.

\section{Gráfico de barras sobre la encuesta de satisfacción a los psicólogos}

Se observa en el siguiente gráfico que las máximas puntuaciones, que se pretende obtener respecto al acuerdo con las preguntas de la encuesta, serán para las preguntas positivas y que la mínima puntuación que se pretende obtener respecto al desacuerdo con las preguntas de la encuesta, serán para las preguntas negativas.

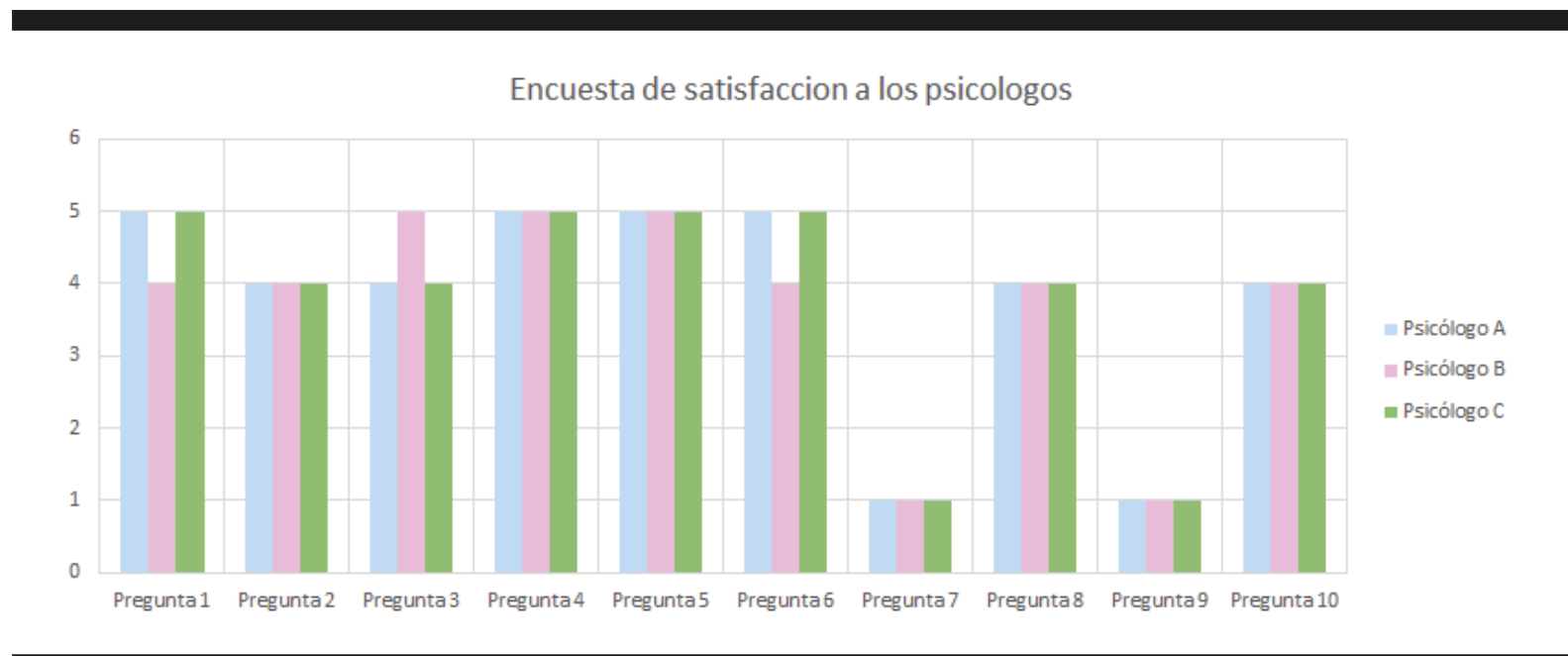

Fig. 2. Gráfico de barras de la satisfacción del psicólogo. Fuente: elaboración propia. 


\section{Encuesta de satisfacción del sistema al paciente}

Como se puede ver en la siguiente tabla se pretende realizar la encuesta a diez pacientes de la clínica COPSI, y observar que la mayoría de ellos se encontrarán satisfechos con el sistema, ya que dan las máximas puntuaciones a las preguntas positivas de la encuesta, es decir que se encuentran de acuerdo con dichas preguntas, como por ejemplo la pregunta 10 y la mínima puntuación a las preguntas negativas de la encuesta, es decir que están en desacuerdo.

Tabla 7. Resultados de la encuesta a los pacientes

\begin{tabular}{|c|c|c|c|c|c|c|c|c|c|c|}
\hline Preguntas de la encuesta & A & $\mathrm{B}$ & $\mathrm{C}$ & $\mathrm{D}$ & $\mathrm{E}$ & $\mathrm{F}$ & G & $\mathrm{H}$ & I & $\mathrm{J}$ \\
\hline 1. Es muy fácil de usar el sistema de evaluación del test BAI & 5 & 4 & 5 & 4 & 4 & 4 & 5 & 5 & 5 & 5 \\
\hline 2. Prefiero utilizar este método de evaluación, que el método manual & 4 & 4 & 4 & 4 & 4 & 4 & 5 & 5 & 5 & 5 \\
\hline 3. Los mensajes que aparece en el sistema son de fácil comprensión & 4 & 5 & 4 & 4 & 4 & 4 & 4 & 4 & 4 & 4 \\
\hline 4. Tuve problemas en utilizar el sistema & 1 & 2 & 2 & 2 & 1 & 2 & 2 & 2 & 2 & 1 \\
\hline $\begin{array}{l}\text { 5. Es muy bueno este método de evaluación, ya que obtengo rápido mi } \\
\text { diagnóstico del Test de BAI }\end{array}$ & 5 & 5 & 5 & 5 & 4 & 4 & 5 & 5 & 5 & 5 \\
\hline 6. Puedo encontrar fácilmente las funciones que ofrece el sistema & 5 & 4 & 5 & 4 & 4 & 4 & 4 & 4 & 4 & 4 \\
\hline $\begin{array}{l}\text { 7. La presentación del contenido es adecuada (tipo y tamaño de la letra, uso } \\
\text { del color, disposición de elementos según su significado, etc.) }\end{array}$ & 1 & 1 & 1 & 4 & 4 & 5 & 4 & 5 & 5 & 5 \\
\hline 8. Necesito del apoyo de un experto para recorrer el sistema & 4 & 4 & 4 & 1 & 1 & 2 & 2 & 2 & 1 & 2 \\
\hline $\begin{array}{l}\text { 9. Se lo recomendaría a alguien, que desee tomar el test de BAI, asistir a la } \\
\text { clínica COPSI, por su nuevo método de evaluación }\end{array}$ & 1 & 1 & 1 & 4 & 5 & 4 & 5 & 5 & 5 & 5 \\
\hline 10. Estoy satisfecho con el sistema. & 4 & 4 & 4 & 5 & 5 & 5 & 5 & 5 & 5 & 5 \\
\hline
\end{tabular}

Fuente: elaboración propia.

\section{Gráfico circular sobre la pregunta 10 de la encuesta de satisfacción a los pacientes}

En el siguiente gráfico circular se representa lo que sería las respuesta con respecto a la pregunta 10 "Estoy satisfecho con el sistema", donde se pretende apreciar que el $70 \%$ de los encuestados se encuentran en total acuerdo con la pregunta, es decir, se sienten satisfechos con el sistema. 


\section{Pregunta 10 : Estoy satisfecho con el sistema}

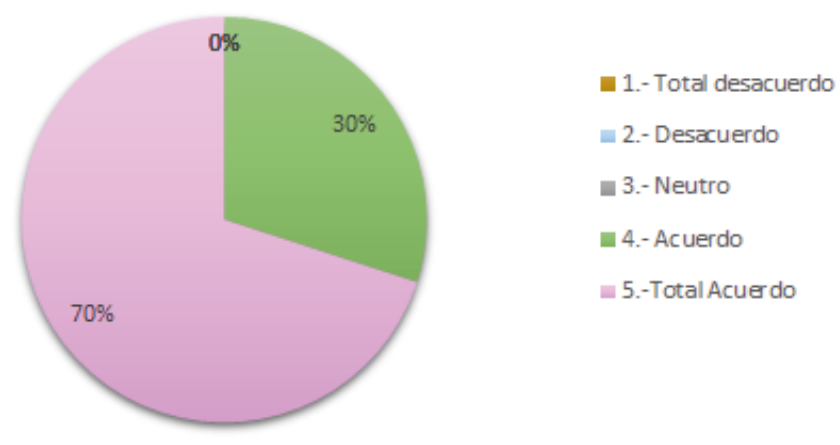

Fig. 3. Gráfico circular de la satisfacción de los pacientes. Fuente: elaboración propia.

\section{Objetivo}

Aumentar la frecuencia con la que se aplica el test psicológico BAI en la clínica psicológica.

\section{Indicador}

Número de pacientes que tomaron el test BAI durante 1 mes.

Validación del aumento de frecuencia de la aplicación del test psicológico BAI

Para comprobar que con la ayuda del sistema experto, permite aumentar la frecuencia con la que se aplica el test psicológico, se realizará un análisis comparativo del registro de pacientes que desean tomar el test BAI, durante un mes en la clínica COPSI.

\section{Resultado}

Se pretende obtener que entre los meses de noviembre y diciembre habrá un aumento de la cantidad de pacientes que tomarán el test psicológico BAI, esto debido a que la clínica ahora ofrecerá una forma más rápida de obtener el diagnóstico del test BAI, a través del sistema experto.

\section{Gráfico de líneas del número de pacientes durante los meses de noviembre y diciembre}

De acuerdo al siguiente gráfico, se pretende obtener que habrá un aumento de pacientes para la evaluación psicológica del test BAI, durante los dos últimos meses del 2019. 


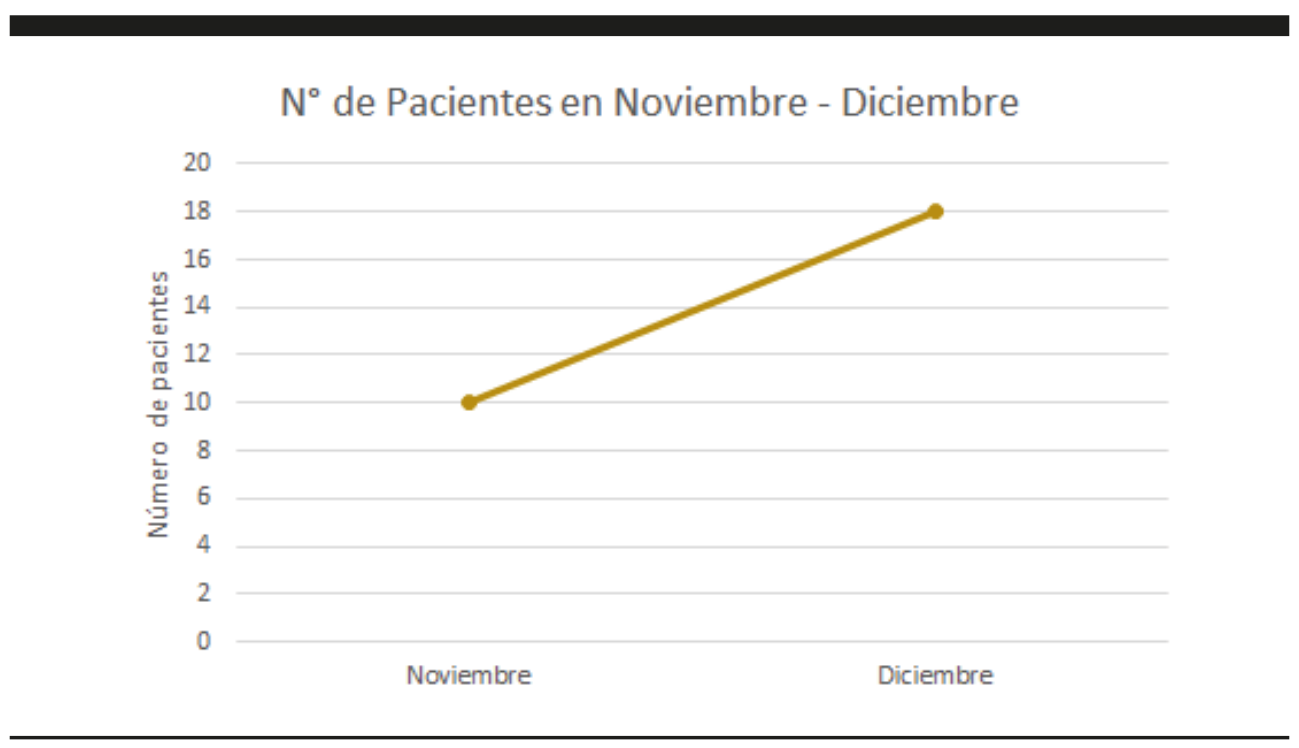

Fig. 4. Gráfico lineal del incremento de los pacientes. Fuente: elaboración propia.

\section{CONCLUSIONES}

Se realizó la evaluación de la clínica COPSI con el objetivo de identificar los procesos y las tareas que comprenden el proceso de la evaluación psicológica de la prueba BAI, lo cual se elaboró el análisis y diseño usando la metodología CommonKADS.

Se identificó los mecanismos de evaluación para medir el nivel de ansiedad y obtener el diagnóstico correcto a través de la evaluación psicológica - Test BAI que busca evaluar el nivel de ansiedad de las personas.

Se desarrolló el análisis, diseño, implementación y el plan de validación del sistema experto a fin de validar su funcionamiento y factibilidad en la clínica COPSI, pretendiendo presentar un $90 \%$ de confiabilidad del SE.

Se presente reducir el tiempo del análisis de las respuestas del test psicológico y la obtención del diagnóstico con respecto al desarrollo manual.

Se pretende aumentar la frecuencia con la que se aplica el test psicológico BAI en la clínica psicológica.

\section{REFERENCIAS BIBLIOGRÁFICAS}

[1] SanaMente. ¿Qué es la ansiedad? [Consultado: 8 de noviembre del 2019]. Disponible en: $<\mathrm{https}$ ://www.sanamente.org/retos/que-es-la-ansiedad/>.

[2] Clínica Las Condes. Inventario de Ansiedad de Beck (BAI). [Consultado: 8 de noviembre del 2019]. Disponible en: <https://www.clinicalascondes.cl/CENTROS-Y-ESPECIALIDADES/ Especialidades/Departamento-de-Medicina-Interna/Unidad-de-Geriatria/Inventario-de-Ansiedad-de-Beck>. 
[3] Badaró, S, Ibañez LJ, Agüero MJ. Sistemas Expertos: Fundamentos, Metodologías y Aplicaciones. Ciencia y Tecnología. 2013;13:349-64.

[4] Barturen Sánchez LY. Desarrollo de un sistema experto sobre web para un diagnóstico temprano de cáncer de cuello uterino en la Clínica Maternidad Belén, Chiclayo (tesis de pregrado). 2012. Disponible en: $<$ http://tesis.usat.edu.pe/handle/usat/519>.

[5] Giraldo, L, Montoya D. Aplicación de la metodología de Commonkads en la gestión del conocimiento. Revista CEA. 2015;1(2):99-108. 